\title{
Comorbidity, age of onset and suicidality in obsessive-compulsive disorder (OCD): An international collaboration ${ }^{2}$, 弥岤
}

\author{
V. Brakoulias ${ }^{\mathrm{a}, *}$, V. Starcevic ${ }^{\mathrm{a}}$, A. Belloch ${ }^{\mathrm{b}}$, C. Brown ${ }^{\text {c }}$, Y.A. Ferrao ${ }^{\mathrm{d}}$, L.F. Fontenelle ${ }^{\mathrm{e}, \mathrm{f}}$, \\ C. Lochner ${ }^{\mathrm{g}}$, D. Marazziti ${ }^{\mathrm{h}}$, H. Matsunaga ${ }^{\mathrm{i}}$, E.C. Miguel ${ }^{\mathrm{j}}$, Y.C.J. Reddy ${ }^{\mathrm{k}}$, M.C. do Rosario ${ }^{1}$, \\ R.G. Shavitt ${ }^{\mathrm{j}}$, A. Shyam Sundar ${ }^{\mathrm{k}}$, D.J. Stein ${ }^{\mathrm{g}}$, A.R. Torres ${ }^{\mathrm{m}}$, K. Viswasam ${ }^{\mathrm{a}}$
}

${ }^{a}$ Department of Psychiatry, The University of Sydney and Nepean Hospital, Sydney Medical School-Nepean, Discipline of Psychiatry, Sydney/Penrith, NSW, Australia ${ }^{\mathrm{b}}$ Department of Personality Psychology, Research Unit for Obsessive-Compulsive and Related Disorders, I'TOC, Faculty of Psychology, Universidad de Valencia, Spain ${ }^{\mathrm{c}}$ NHMRC Clinical Trials Centre, the University of Sydney, Sydney, NSW, Australia

${ }^{\mathrm{d}}$ Department of Psychiatry, Federal University of Health Sciences of Porto Alegre, Brazil

${ }^{\mathrm{e}}$ Department of Psychiatry and Legal Medicine, Federal University of Rio de Janeiro, D'Or Institute for Research and Education, Brazil ${ }^{\mathrm{f}}$ Monash Institute of Cognitive and Clinical Neurosciences, Monash University

${ }^{\mathrm{g}}$ MRC Unit on Anxiety Disorders, Department of Psychiatry, University of Stellenbosch, Cape Town, South Africa

${ }^{\mathrm{h}}$ Dipartimento di Medicina Clinica e Sperimentale, Section of Psychiatry, Dipartmento di Farmacia, Univeristy of Pisa, Italy iDepartment of Neuropsychiatry, Hyogo College of Medicine, Hyogo, Japan

${ }^{\mathrm{j} D e p a r t m e n t}$ and Institute of Psychiatry, Faculty of Medicine, University of São Paulo, Brazil

${ }^{\mathrm{k}}$ Department of Psychiatry National Institute of Mental Health and Neurosciences (NIMHANS), Bengaluru, India

${ }^{1}$ Child and Adolescent Psychiatry Unit (UPIA) at the Department of Psychiatry, Federal University of São Paulo (UNIFESP), Brazil

${ }^{\mathrm{m}}$ Department of Neurology, Psychology and Psychiatry, Botucatu Medical School, Univ Estadual Paulista (UNESP), Brazil

\begin{abstract}
Objectives: To collate data from multiple obsessive-compulsive disorder (OCD) treatment centers across seven countries and five continents, and to report findings in relation to OCD comorbidity, age of onset of OCD and comorbid disorders, and suicidality, in a large clinical and ethnically diverse sample, with the aim of investigating cultural variation and the utility of the psychiatric diagnostic classification of obsessive-compulsive and related disorders.

Methods: Researchers in the field of OCD were invited to contribute summary statistics on current and lifetime psychiatric comorbidity, age of onset of OCD and comorbid disorders and suicidality in their patients with OCD.

Results: Data from 3711 adult patients with primary OCD came from Brazil $(\mathrm{n}=955)$, India $(\mathrm{n}=802)$, Italy (n=750), South Africa $(\mathrm{n}=565)$, Japan $(\mathrm{n}=322)$, Australia $(\mathrm{n}=219)$, and Spain $(\mathrm{n}=98)$. The most common current comorbid disorders were major depressive disorder $(28.4 \% ; \mathrm{n}=1055)$, obsessive-compulsive personality disorder $(24.5 \%, \mathrm{n}=478)$, generalized anxiety disorder $(19.3 \%$, $\mathrm{n}=716)$, specific phobia $(19.2 \%, \mathrm{n}=714)$ and social phobia $(18.5 \%, \mathrm{n}=686)$. Major depression was also the most commonly co-occurring lifetime diagnosis, with a rate of $50.5 \%(\mathrm{n}=1874)$. OCD generally had an age of onset in late adolescence $($ mean $=17.9$ years, SD $=1.9)$. Social phobia, specific phobia and body dysmorphic disorder also had an early age of onset. Co-occurring major depressive disorder, generalized anxiety disorder and psychotic disorders tended to have a later age of onset than OCD. Suicidal ideation within the last month was reported by $6.4 \%(n=200)$ of patients with OCD and 9.0\% $(n=314)$ reported a lifetime history of suicide attempt.
\end{abstract}

\footnotetext{
is Conflict of interest: No conflicts of interest.

败败 Sources of funding: Nepean Medical Research Foundation, Pfizer Neuroscience Grant Programme, Spanish MINECO Grant PSI2013-44733-R, and The University of Sydney.

* Corresponding author at: Nepean Hospital, Department of Psychiatry, PO Box 63, Penrith, NSW 2751, Australia. Tel.: +61 247342585 ; fax: +61 24734 3343.

E-mail addresses: vbrakoulias@bigpond.com, vlasios.brakoulias@sydney.edu.au (V. Brakoulias).
} 
Conclusions: In this large cross-continental study, comorbidity in OCD was common. The high rates of comorbid major depression and anxiety disorders emphasize the need for clinicians to assess and monitor for these disorders. Earlier ages of onset of OCD, specific phobia and social phobia may indicate some relatedness between these disorders, but this requires further study. Although there do not appear to be significant cultural variations in rates or patterns of comorbidity and suicidality, further research using similar recruitment strategies and controlling for demographic and clinical variables may help to determine whether any sociocultural factors protect against suicidal ideation or psychiatric comorbidity in patients with OCD.

(C) 2017 Elsevier Inc. All rights reserved.

\section{Introduction}

Obsessive-compulsive disorder (OCD) is a distressing and disabling disorder characterized by repetitive and intrusive thoughts, urges, images or fears (obsessions) and repetitive behaviors or mental acts (compulsions). Common symptoms include fears of contamination and excessive hand washing, preoccupation with symmetry and ordering, intrusive and/or distressing unacceptable or taboo thoughts and repetitive checking [1]. OCD is thought to occur in $0.8 \%$ to $2 \%$ of the population [2]. OCD is associated with high rates of comorbidity with other psychiatric disorders in clinical samples, with several studies reporting rates of at least one other co-occurring psychiatric disorder ranging from $65 \%$ in India [3] and $69 \%$ in Turkey [4] to as high as $92 \%$ in Brazil [5]. In a recent international study of OCD comorbidity, limited by a small sample size, the most common co-occurring psychiatric diagnoses were major depressive disorder and the anxiety disorders [6].

In an attempt to better understand the nature of the relationship between OCD and other psychiatric disorders, some studies have assessed the age of onset of the co-occurring disorders. These studies reported that anxiety, mood, eating and tic disorders tended to have early ages of onset [7] and that the age of onset of separation anxiety disorder, attention deficit hyperactivity disorder and tic disorders preceded the onset of OCD [8]. Age of onset of comorbid psychiatric disorders in OCD may be influenced by socio-cultural factors in addition to genetic factors. Hence, investigating whether age of onset differs internationally may give clues as to whether specific social or cultural practices in different countries may precipitate OCD.

OCD has in the past, been considered a psychiatric disorder with relatively low rates of suicidal ideation, however a recent study [9] concluded that the risk of attempted and completed suicide is substantial among individuals with OCD. Some authors suggest that suicide risk is more strongly predicted by psychiatric comorbidity, e.g. rates of comorbid depression and anxiety rather than OCD itself $[10,11]$, but controlling for comorbidity the risk of suicidality remains substantial among OCD sufferers (de la Cruz et al., 2016). In general, studies have reported high rates of suicidal ideation in OCD ranging between $20 \%$ and $46 \%$ [11-15]. Another recent study reported that $65 \%$ of the OCD patients had endorsed at least one of the questions about suicidal phenomena as positive. Of these, $32 \%$ had made suicidal plans, and $19.4 \%$ had attempted suicide [16].
Links have also been reported between suicidal ideation and alexithymia in patients with OCD [17].

This study aimed to assess comorbidity, age of onset and suicidality in a large international study. Drawing from existing literature, we hypothesized that patients with OCD would have high rates of psychiatric comorbidity, that rates would vary significantly between countries, and that major depressive disorder and anxiety disorders would be the most common comorbid psychiatric disorders. We also hypothesized that the age of onset of specific phobia and social phobia would precede the age of onset to OCD and that rates of suicidal ideation would be between $20 \%$ and $50 \%$ based on previous studies.

\section{Methods}

The author VB wrote to leading international OCD researchers with the aim of having at least one sample from each of the five inhabited continents of the globe represented in a large international survey. Researchers from specialized centers in the seven countries (Australia, Brazil, India, Italy, Japan, South Africa and Spain) were asked to complete a standardized data collection sheet using data from their studies, e.g. [18]. All but one center agreed to participate. Individual studies were approved by their respective Institutional Review Boards. The survey assessed current and lifetime psychiatric comorbidity, suicidal ideation within the last month and any previous suicide attempt, and the age of onset of comorbid disorders. Assessment occurred cross-sectionally, upon referral to each research center. Information was collected regarding the size of the sample, the years in which the sample was assessed, the mean age and gender distribution of the sample, the mean severity of OCD in the sample, and the mode of referral of participants to the study.

The severity of OCD was assessed with the Yale-Brown Obsessive Compulsive Scale (Y-BOCS) [19] in all samples and diagnosis of OCD was made with the Structured Clinical Interview for DSM-IV-TR Axis I disorders (SCID) [20], the Mini International Neuropsychiatric Interview plus version (MINI [21,22]), or the Anxiety Disorder Interview Schedule for DSM-IV Lifetime version (ADIS) [23].

Consistency of summary statistics across countries was assessed using chi-square tests for categorical variables (i.e. gender, referral type, comorbid disorders, suicidal ideation), and the $\mathrm{Q}$ heterogeneity statistic for continuous variables (i.e. 
age, YBOCS score). Overall summary estimates were computed using inverse variance weighting. Analyses were performed in SAS version 9.3 (SAS Institute).

\section{Results}

The sample came from Australia, Brazil, India, Italy, Japan, South Africa and Spain and had a total of 3711 participants. The characteristics of the seven international samples are shown in Table 1. Although the data were collected in the same 10 to 15 year period, participants varied significantly in age (for example, the sample from Australia was on average 14 years older than the sample from India) (overall p-value for heterogeneity in age distribution across countries was $p=0.99)$. OCD severity also differed significantly across sites, with the mean Y-BOCS score of the Italian sample (mean score 30.0 ( $\mathrm{SD}=8.0$ ); severe range) nearly 10 points higher than that of the South African sample (mean score 20.4 ( $\mathrm{SD}=7.2$ ); moderate range) (overall heterogeneity $p=0.97$ ). There was variation in the manner by which participants were referred to each center, with most participants in the samples from Japan ( $\mathrm{n}=292 ; 90.7 \%$ ) referred by a doctor, whereas the majority of the participants from the sample from India ( $\mathrm{n}=$ $547 ; 68.2 \%$ ) were self-referred (heterogeneity $\mathrm{p}<0.001$ ).

Current psychiatric comorbidity is shown in Table 2. The most commonly co-occurring diagnoses were major depressive disorder $(28.4 \%, \mathrm{n}=1055)$, obsessive-compulsive personality disorder (OCPD) $(24.8 \%, \mathrm{n}=478)$, generalized anxiety disorder $(19.3 \%, \mathrm{n}=716)$, specific phobia $(19.2 \%$, $\mathrm{n}=714)$ and social phobia $(18.5 \%, \mathrm{n}=686)$. Hoarding symptoms also commonly co-occurred at a rate of $23.4 \%$ $(n=507)$, however assessment of hoarding disorder was not standardized (criteria for hoarding disorder were only published in 2013 and all of these studies had started before this). The South African $(17.5 \%, \mathrm{n}=99)$ and the Australian $(18.2 \%, \mathrm{n}=39)$ samples had relatively lower rates of co-occurring major depression. The Australian sample had a higher rate of co-occurring OCPD (50.5\%, $\mathrm{n}=108)$, whereas OCPD was not assessed in the Brazilian and Indian samples. Rates of generalized anxiety disorder were higher in the Brazilian (33.6\%, $\mathrm{n}=321)$, Australian $(30.8 \%, \mathrm{n}=66)$ and Italian $(24.0 \%, \mathrm{n}=180)$ samples. Specific phobia was more often comorbid in the Brazilian $(31.0 \%, \mathrm{n}=296)$ and Italian $(30.8 \%, \mathrm{n}=231)$ samples, and social phobia was more often comorbid in the Brazilian $(32.7 \%, \mathrm{n}=312)$ sample compared to other samples.

Lifetime comorbidity is shown in Table 3. The most common lifetime diagnoses reported were major depressive disorder $(50.5 \%, \mathrm{n}=1874)$, social phobia $(26.4 \%, \mathrm{n}=768)$, specific phobia $(25.5 \%, \mathrm{n}=948)$, generalized anxiety disorder $(24.0 \%, \mathrm{n}=890)$, hypomanic episode $(23.7 \%$, $\mathrm{n}=746)$ and dysthymia $(22.5 \%, \mathrm{n}=654)$. It should be noted that the mean rate of a lifetime diagnosis of hypomania was raised by a significantly higher rate of a lifetime diagnosis of hypomania in the Italian $(92.3 \%, \mathrm{n}=672)$ sample. The Indian $(32.3 \%, \mathrm{n}=259)$ and Japanese $(36.6 \%$, $\mathrm{n}=118$ ) samples had relatively lower rates of a lifetime diagnosis of major depressive disorder compared to the other samples. A lifetime diagnosis of specific phobia was lower in the Indian $(1.1 \%, \mathrm{n}=9)$ sample and lifetime generalized anxiety disorder was more frequent in the Italian sample $(48.0 \%, \mathrm{n}=360)$ compared to the other samples.

The ages of onset of OCD and of comorbid psychiatric disorders are shown in Table 4 and Fig. 1. Data were available for only 4 centers. The mean age of onset of OCD was 16.9 years $(\mathrm{SD}=4.5)$. Co-occurring specific phobia $($ mean $=16.6$ years, $\mathrm{SD}=2.1)$ and social phobia $($ mean $=$ 17.3 years, $\mathrm{SD}=2.0$ ) had a similar age of onset, whereas

Table 1

Characteristics of the seven international samples $(\mathrm{N}=3711)$.

\begin{tabular}{|c|c|c|c|c|c|c|c|c|c|}
\hline & $\begin{array}{l}\text { Sample } \\
\text { Size n (\%) }\end{array}$ & $\begin{array}{l}\text { Proportion } \\
\text { Male n (\%) }\end{array}$ & $\begin{array}{l}\text { Mean Age } \\
\text { (years) } \\
\text { M(SD) }\end{array}$ & $\begin{array}{l}\text { Mean Total } \\
\text { Y-BOCS } \\
\text { M (SD) }\end{array}$ & $\begin{array}{l}\text { Referral by a } \\
\text { doctor } \mathrm{n}(\%)\end{array}$ & $\begin{array}{l}\text { Referral by other } \\
\text { health professional } \\
\mathrm{n}(\%)\end{array}$ & $\begin{array}{l}\text { Self-referral } \\
\mathrm{n}(\%)\end{array}$ & $\begin{array}{l}\text { Method of } \\
\text { diagnosis }\end{array}$ & $\begin{array}{l}\text { Years of data } \\
\text { collection }\end{array}$ \\
\hline Australia & $219(5.9)$ & $90(41.3)$ & $44.1(16.0)$ & $22.3(7.0)$ & 121 & $20(9.1)$ & $70(32.0)$ & MINI & 2007 to 2015 \\
\hline Brazil & $955(25.7)$ & $400(41.9)$ & $35.8(12.5)$ & $25.6(7.4)$ & a & $\mathrm{a}$ & a & SCID & 2003 to 2009 \\
\hline India & $802(21.6)$ & $497(62.0)$ & $29.4(10.4)$ & $23.5(8.0)$ & $206(25.7)$ & NA & $547(68.2)$ & MINI & 2004 to 2012 \\
\hline Italy & $750(20.2)$ & $368(49.1)$ & $39.0(11.0)$ & $30.0(8.0)$ & $250(33.3)$ & $230(30.7)$ & $270(36.0)$ & SCID & 2008 to 2014 \\
\hline Japan & $322(8.7)$ & $139(43.2)$ & $31.9(9.2)$ & $27.3(4.9)$ & $292(90.7)$ & $10(3.1)$ & $20(6.2)$ & SCID & 2007 to 2013 \\
\hline South Africa & $565(15.2)$ & $282(49.9)$ & $32.2(13.0)$ & $20.4(7.2)$ & $172(30.4)$ & $0(0.0)$ & $393(69.6)$ & SCID or MINI & 2000 to 2016 \\
\hline $\begin{array}{l}\text { Spain } \\
\text { p-value }\end{array}$ & $98(2.6)$ & $\begin{array}{l}48(49.0) \\
<0.001^{\mathrm{b}}\end{array}$ & $\begin{array}{l}33.8(11.3) \\
0.99^{\text {cd }}\end{array}$ & $\begin{array}{l}27.1(6.0) \\
0.97^{\mathrm{c}}\end{array}$ & $64(65.3)$ & $\begin{array}{l}9(9.2) \\
<0.001^{b}\end{array}$ & $25(25.5)$ & ADIS-IV-L & 2013 to 2016 \\
\hline Total survey sample & 3711 & $1824(49.2)$ & $35.2(11.9)$ & $25.2(6.9)$ & $1105(40.1)$ & $269(13.8)$ & $1325(48.1)$ & N/A & 2000 to 2016 \\
\hline
\end{tabular}

ADIS-IV-L = Anxiety Disorders Interview Schedule-IV Lifetime, MINI = MINI Neuropsychiatric diagnostic assessment, NA $=$ Not assessed, N/A $=$ Not applicable, SCID = Structured Clinical Interview for DSM IV-TR, Y-BOCS = Yale-Brown Obsessive-Compulsive Scale.

a Referral source was not specified for $60 \%$ of the Brazilian sample.

b Chi-square test.

c $\mathrm{Q}$ test for heterogeneity.

${ }^{\mathrm{d}}$ Chi-square test of referral type (doctor/other health professional/self) by country. Percentages have been calculated using a total sample size of 2184 as the Brazilian sample was excluded. 
Table 2

Rates of co-occurring psychiatric diagnoses, hoarding symptoms and suicidal ideation at the time of assessment across the samples from seven nations $(\mathrm{N}=$ 3711).

\begin{tabular}{|c|c|c|c|c|c|c|c|c|c|c|c|c|c|c|c|c|c|}
\hline & \multicolumn{2}{|c|}{$\begin{array}{l}\text { Australia } \\
(\mathrm{N}=219)\end{array}$} & \multicolumn{2}{|c|}{$\begin{array}{c}\text { Brazil } \\
(\mathrm{N}=955)\end{array}$} & \multicolumn{2}{|c|}{$\begin{array}{c}\text { India } \\
(\mathrm{N}=802)\end{array}$} & \multicolumn{2}{|c|}{$\begin{array}{c}\text { Italy }^{\mathrm{a}} \\
(\mathrm{N}=750)\end{array}$} & \multicolumn{2}{|c|}{$\begin{array}{c}\text { Japan } \\
(\mathrm{N}=322)\end{array}$} & \multicolumn{2}{|c|}{$\begin{array}{c}\text { South } \\
\text { Africa } \\
(\mathrm{N}=565) \\
\end{array}$} & \multicolumn{2}{|c|}{$\begin{array}{c}\text { Spain } \\
(\mathrm{N}=98)\end{array}$} & \multicolumn{2}{|c|}{$\begin{array}{l}\text { All nations } \\
(\mathrm{N}=3711)\end{array}$} & \multirow{2}{*}{$\frac{\text { p-value }}{\text { Chi-squared }}$} \\
\hline & $\mathrm{N}$ & $\%$ & $\mathrm{~N}$ & $\%$ & $\mathrm{~N}$ & $\%$ & $\mathrm{~N}$ & $\%$ & $\mathrm{~N}$ & $\%$ & $\mathrm{~N}$ & $\%$ & $\mathrm{~N} \%$ & & $\mathrm{~N}$ & $\%$ & \\
\hline Major depressive disorder & 39 & 18.2 & 321 & 33.6 & 220 & 27.4 & 240 & 32.0 & 90 & 28.0 & 99 & 17.5 & 46 & 46.9 & 1055 & 28.4 & $<\mathbf{0 . 0 0 1}$ \\
\hline Dysthymia & 18 & 8.4 & 110 & 11.5 & 131 & 16.3 & 189 & 25.2 & 26 & 8.1 & 82 & 14.5 & 8 & 8.2 & 564 & 15.2 & $<0.001$ \\
\hline Manic episode & $\mathrm{X}$ & $X$ & 4 & 0.4 & 6 & 0.7 & 27 & 3.6 & 6 & 1.9 & 13 & 2.3 & 4 & 4.1 & 60 & 1.7 & $<\mathbf{0 . 0 0 1}$ \\
\hline Hypomanic episode & $\mathrm{X}$ & $\mathrm{X}$ & 13 & 1.4 & 10 & 1.2 & 280 & 37.3 & 11 & 3.4 & $\mathrm{Y}$ & $\mathrm{Y}$ & 2 & 2.0 & 316 & 10.8 & $<\mathbf{0 . 0 0 1}$ \\
\hline Panic disorder & 33 & 15.4 & 44 & 4.6 & 30 & 3.7 & 67 & 8.9 & 29 & 9.0 & 42 & 7.5 & 8 & 8.2 & 253 & 6.8 & $<\mathbf{0 . 0 0 1}$ \\
\hline Agoraphobia & 29 & 13.6 & 45 & 4.7 & 6 & 0.7 & 53 & 7.1 & 32 & 9.9 & 2 & 0.4 & 4 & 4.1 & 171 & 4.6 & $<\mathbf{0 . 0 0 1}$ \\
\hline Social phobia & 38 & 17.8 & 312 & 32.7 & 101 & 12.6 & 120 & 16.0 & 58 & 18.0 & 48 & 8.5 & 9 & 9.2 & 686 & 18.5 & $<\mathbf{0 . 0 0 1}$ \\
\hline Posttraumatic stress disorder & 20 & 9.3 & 97 & 10.2 & 0 & 0.0 & 0 & 0.0 & 13 & 4.0 & 7 & 1.2 & 1 & 1.0 & 138 & 3.7 & $<\mathbf{0 . 0 0 1}$ \\
\hline Generalized anxiety disorder & 66 & 30.8 & 321 & 33.6 & 51 & 6.4 & 180 & 24.0 & 29 & 9.0 & 60 & 10.6 & 9 & 9.2 & 716 & 19.3 & $<\mathbf{0 . 0 0 1}$ \\
\hline Specific phobia & 42 & 19.6 & 296 & 31.0 & 9 & 1.1 & 231 & 30.8 & 59 & 18.3 & 73 & 12.9 & 4 & 4.1 & 714 & 19.2 & $<\mathbf{0 . 0 0 1}$ \\
\hline Alcohol abuse & 14 & 6.5 & $\mathrm{X}$ & $\mathrm{X}$ & $\mathrm{b}$ & $\mathrm{b}$ & 20 & 2.7 & 17 & 5.3 & 14 & 2.5 & 2 & 2.0 & 67 & 3.4 & $<\mathbf{0 . 0 0 1}$ \\
\hline Alcohol dependence & $\mathrm{X}$ & $\mathrm{X}$ & $\mathrm{X}$ & $\mathrm{X}$ & 6 & 0.7 & 3 & 0.4 & 13 & 4.0 & 5 & 0.9 & $\mathrm{X}$ & $\mathrm{X}$ & 27 & 1.1 & $<\mathbf{0 . 0 0 1}$ \\
\hline Non-alcohol drug abuse & 2 & 0.9 & 12 & 1.3 & $\mathrm{~b}$ & $\mathrm{~b}$ & 5 & 0.7 & 39 & 12.1 & 4 & 0.7 & $\mathrm{X}$ & $\mathrm{X}$ & 62 & 2.2 & $<\mathbf{0 . 0 0 1}$ \\
\hline Non-alcohol drug dependence & $\mathrm{X}$ & $\mathrm{X}$ & 9 & 0.9 & 25 & 3.1 & 5 & 0.7 & 23 & 7.1 & 3 & 0.5 & $\mathrm{X}$ & $X$ & 65 & 1.9 & $<\mathbf{0 . 0 0 1}$ \\
\hline Psychotic disorder & $\mathrm{X}$ & $X$ & $\mathrm{X}$ & $X$ & 22 & 2.7 & 47 & 6.3 & 6 & 1.9 & 0 & 0.0 & $\mathrm{X}$ & $X$ & 78 & 2.2 & $<0.001$ \\
\hline Hypochondriasis & 5 & 2.3 & 1 & .1 & 8 & 1 & 61 & 8.1 & 26 & 8.1 & 9 & 1.6 & 12 & 12.2 & 122 & 3.3 & $<\mathbf{0 . 0 0 1}$ \\
\hline Anorexia nervosa & 0 & 0.0 & 31 & 3.2 & 0 & 0 & 43 & 5.7 & 10 & 3.1 & 4 & 0.7 & 1 & 1.0 & 89 & 2.4 & $<\mathbf{0 . 0 0 1}$ \\
\hline Bulimia nervosa & 2 & 0.9 & 7 & .7 & 0 & 0 & 28 & 3.7 & 19 & 5.9 & 7 & 1.2 & 2 & 2.0 & 65 & 1.8 & $<\mathbf{0 . 0 0 1}$ \\
\hline Body dysmorphic disorder & 6 & 2.8 & 16 & 1.7 & 5 & 0.6 & 97 & 12.9 & 39 & 12.1 & 34 & 6.0 & 4 & 4.1 & 201 & 5.4 & $<\mathbf{0 . 0 0 1}$ \\
\hline $\begin{array}{c}\text { Obsessive-compulsive } \\
\text { personality disorder }\end{array}$ & 108 & 50.5 & $\mathrm{Y}$ & Y & Y & $\mathrm{Y}$ & 167 & 22.3 & 45 & 14.0 & 150 & 26.5 & 8 & 8.2 & 478 & 24.5 & $<\mathbf{0 . 0 0 1}$ \\
\hline Tic Disorder & 26 & 11.3 & 264 & 27.6 & 33 & 4.1 & 77 & 10.3 & $12^{\mathrm{b}}$ & $13.6^{\mathrm{b}}$ & $49^{c}$ & $9.1^{\mathrm{c}}$ & 0 & 0 & 461 & 13.4 & $<0.001$ \\
\hline Trichotillomania & 45 & 5.0 & 45 & 4.7 & $\mathrm{Y}$ & $\mathrm{Y}$ & 50 & 6.7 & $11^{\mathrm{b}}$ & $12.5^{\mathrm{b}}$ & 14 & 2.5 & 1 & 1.1 & 166 & 6.2 & $<\mathbf{0 . 0 0 1}$ \\
\hline Skin-picking disorder & 54 & 24.7 & 146 & 15.3 & $\mathrm{Y}$ & $\mathrm{Y}$ & 7 & 0.9 & $12^{b}$ & $13.6^{\mathrm{b}}$ & $9^{c}$ & $1.6^{\mathrm{c}}$ & 1 & 1.0 & 229 & 7.9 & $<\mathbf{0 . 0 0 1}$ \\
\hline Hoarding symptoms & 46 & 20.7 & 421 & 44.1 & Y & $\mathrm{Y}$ & 24 & 3.2 & $10^{\mathrm{b}}$ & $11.4^{\mathrm{b}}$ & $4^{c}$ & $0.7^{\mathrm{c}}$ & $2^{e}$ & $2.1^{\mathrm{d}}$ & 507 & 23.4 & $<0.001$ \\
\hline Suicidal ideation within last month & 71 & 33.2 & 100 & 10.5 & 11 & 1.4 & 0 & 0.0 & 16 & 5.0 & $\mathrm{Y}$ & $\mathrm{Y}$ & 2 & 2.0 & 200 & 6.4 & $<\mathbf{0 . 0 0 1}$ \\
\hline
\end{tabular}

$\mathrm{X}=$ Study exclusion criteria prevented participants having this co-occurring diagnosis. $\mathrm{Y}=$ Not assessed with diagnostic interview in this particular study.

a The Italian center was the only center that assessed for attention deficit hyperactivity disorder (ADHD). Their sample reported that 4.9\% ( $\mathrm{n}=37$ ) had comorbid ADHD.

b The Japanese sample assessed 88 patients for additional comorbidity. Hence total sample for these comorbidities is 88 . They also assessed for internet gaming disorder $(n=6,7 \%)$, compulsive buying $(n=11,12 \%)$ and olfactory reference syndrome $(n=8,9 \%)$ in this smaller sample.

c The South African assessed on 539 for tics, 287 for skin picking disorder, and 61 for hoarding disorder.

d Obsessive-compulsive disorder was secondary to hoarding disorder in these 2 cases.

major depressive disorder $($ mean $=24.5$ years, $\mathrm{SD}=3.1)$, panic disorder (mean $=24.5$ years, $\mathrm{SD}=3.2$ ) and posttraumatic stress disorder $($ mean $=24.0$ years, $\mathrm{SD}=7.8)$ had a later age of onset (See Fig. 1).

Suicidality is reported in Tables 2 and 3. Suicidal ideation within the last month was reported by a mean of $6.4 \%(n=$ $200)$ of patients, while $9.0 \%(n=314)$ reported having attempted suicide in their lifetime. Recent suicidal ideation was highest in the Australian sample $(33.2 \%, \mathrm{n}=71)$. A lifetime history of a suicide attempt was highest in the South African sample $(15.9 \%, \mathrm{n}=90)$.

\section{Discussion}

This survey of OCD comorbidity in more than 3700 participants across seven countries represents the largest international evaluation of OCD comorbidity in the literature thus far. Consistent with existing literature, OCD has high rates of comorbidity, particularly with major depressive disorder. Rates of lifetime major depressive disorder ranged from around $30 \%$ in India and Japan to around $60 \%$ in Australia and South Africa. Conversely, the rates of a current diagnosis of major depressive disorder in the Australian and South African samples were the lowest at just below $20 \%$. Patients in Australia and South Africa may have had better access to treatment for mood disorders or may have had their major depression treated prior to presenting to an OCD research center. Patients in India and Japan may have had a lower rate of a lifetime diagnosis of major depressive disorder because they were younger, i.e. they had lower mean ages. The mean age of onset of comorbid major depressive disorder was 24.5 years. Regardless of the variations between the centers, the fact that depressive disorders (both major depressive disorder and dysthymic disorder) co-occurred so commonly with OCD suggests an important relationship between OCD and depressive disorders, with possible etiological implications. Also, there are 
Table 3

Rates of co-occurring lifetime diagnoses and suicide attempts across the samples from seven nations $(\mathrm{N}=3711)^{\mathrm{a}}$

\begin{tabular}{|c|c|c|c|c|c|c|c|c|c|c|c|c|c|c|c|c|c|}
\hline & \multicolumn{2}{|c|}{$\begin{array}{l}\text { Australia } \\
(\mathrm{N}=219)\end{array}$} & \multicolumn{2}{|c|}{$\begin{array}{c}\text { Brazil } \\
(\mathrm{N}=955)\end{array}$} & \multicolumn{2}{|c|}{$\begin{array}{c}\text { India } \\
(\mathrm{N}=802)\end{array}$} & \multicolumn{2}{|c|}{$\begin{array}{c}\text { Italy } \\
(\mathrm{N}=750)\end{array}$} & \multicolumn{2}{|c|}{$\begin{array}{c}\text { Japan } \\
(\mathrm{N}=322)\end{array}$} & \multicolumn{2}{|c|}{$\begin{array}{c}\text { South } \\
\text { Africa } \\
(\mathrm{N}=565)\end{array}$} & \multicolumn{2}{|c|}{$\begin{array}{c}\text { Spain } \\
(\mathrm{N}=98)\end{array}$} & \multicolumn{2}{|c|}{$\begin{array}{l}\text { All nations } \\
(\mathrm{N}=3711)\end{array}$} & \multirow{2}{*}{$\begin{array}{c}\text { P-value } \\
\text { chi-squared }\end{array}$} \\
\hline & $\mathrm{N}$ & $\%$ & $\mathrm{~N}$ & $\%$ & $\mathrm{~N}$ & $\%$ & $\mathrm{~N}$ & $\%$ & $\mathrm{~N}$ & $\%$ & $\mathrm{~N}$ & $\%$ & $\mathrm{~N} \%$ & & $\mathrm{~N}$ & $\%$ & \\
\hline Major depressive disorder & 134 & 62.6 & 549 & 57.5 & 259 & 32.3 & 420 & 56.0 & 118 & 36.6 & 340 & 60.2 & 54 & 55.1 & 1874 & 50.5 & $<\mathbf{0 . 0 0 1}$ \\
\hline Dysthymia & 31 & 14.5 & 115 & 12.0 & $\mathrm{Y}$ & $\mathrm{Y}$ & 378 & 25.2 & 39 & 12.1 & 79 & 14.0 & 12 & 12.2 & 654 & 22.5 & $<\mathbf{0 . 0 0 1}$ \\
\hline Manic episode & 8 & 3.7 & 34 & 3.6 & 30 & 3.7 & 54 & 7.2 & 19 & 5.9 & 17 & 3.0 & 6 & 6.1 & 168 & 4.5 & 0.0018 \\
\hline Hypomanic episode & 7 & 3.2 & 33 & 3.5 & 1 & 1 & 672 & 92.3 & 16 & 5.0 & $\mathbf{N A}$ & NA & 3 & 3.1 & 746 & 23.7 & $<\mathbf{0 . 0 0 1}$ \\
\hline Panic disorder & 47 & 22.0 & 59 & 6.2 & 30 & 3 & 134 & 17.9 & 45 & 14.0 & 65 & 11.6 & 28 & 28.6 & 408 & 11.0 & $<0.001$ \\
\hline Agoraphobia & 30 & 14.0 & 48 & 5.0 & 7 & 0.9 & 105 & 14.0 & 55 & 17.1 & 3 & 0.5 & 14 & 14.3 & 262 & 7.1 & $<\mathbf{0 . 0 0 1}$ \\
\hline Social phobia & 52 & 24.3 & 336 & 35.2 & $\mathrm{Y}$ & $\mathrm{Y}$ & 240 & 32.0 & 74 & 23.0 & 47 & 8.3 & 19 & 19.4 & 768 & 26.4 & $<\mathbf{0 . 0 0 1}$ \\
\hline Posttraumatic stress disorder & 28 & 13.1 & 185 & 19.4 & $\mathrm{Y}$ & $\mathrm{Y}$ & 44 & 5.9 & 13 & 4.0 & 24 & 4.2 & 1 & 1.0 & 295 & 10.1 & $<\mathbf{0 . 0 0 1}$ \\
\hline Generalized anxiety disorder & 45 & 21.0 & 326 & 34.1 & 43 & 5.4 & 360 & 48.0 & 29 & 9.0 & 66 & 11.7 & 21 & 21.4 & 890 & $\mathbf{2 4 . 0}$ & $<0.001$ \\
\hline Specific phobia & 33 & 15.4 & 302 & 31.6 & 9 & 1.1 & 462 & 61.6 & 59 & 18.3 & 74 & 13.1 & 9 & 9.2 & 948 & 25.5 & $<\mathbf{0 . 0 0 1}$ \\
\hline Alcohol abuse & 45 & 21.0 & $\mathbf{X}$ & $\mathbf{X}$ & $\mathrm{Y}$ & $\mathrm{Y}$ & 38 & 5.1 & 29 & & 36 & 6.4 & 6 & 6.1 & 154 & 7.9 & $<0.001$ \\
\hline Alcohol dependence & 25 & 11.7 & $\mathbf{X}$ & $\mathbf{X}$ & $\mathrm{Y}$ & Y & 6 & & 16 & & 16 & & $\mathrm{X}$ & $\mathrm{X}$ & 63 & 3.4 & $<\mathbf{0}$. \\
\hline Non-alcol & 1 & 7.9 & 27 & 2.8 & $\mathrm{Y}$ & Y & م & & 4 & & 15 & & 1 & & 116 & 4.0 & $<\mathbf{0 . 0 0 1}$ \\
\hline Non-alcohol drug dependence & 9 & 4.2 & 13 & 1.4 & $\mathrm{Y}$ & Y & 10 & 1.3 & 23 & 7.1 & 10 & 1.8 & $\mathrm{X}$ & $\mathrm{X}$ & 65 & 2.3 & $<\mathbf{0 . 0 0 1}$ \\
\hline Psychotic disorder & 25 & 11.7 & $\mathrm{X}$ & $\mathrm{X}$ & 26 & 3.2 & 94 & 12.5 & 8 & 2.5 & $\mathrm{X}$ & $\mathrm{X}$ & $\mathrm{X}$ & $\mathrm{X}$ & 159 & 4.3 & $<\mathbf{0 . 0 0 1}$ \\
\hline Hypochondriasis & 7 & 3.3 & 33 & 3.5 & $\mathrm{Y}$ & Y & 122 & 16.3 & 30 & 9.3 & 15 & 2.7 & 16 & 16.3 & 223 & 7.7 & $<0.001$ \\
\hline Anorexia nervosa & 5 & 2.3 & 25 & 2.6 & $\mathrm{Y}$ & $\mathrm{Y}$ & 86 & 11.5 & 16 & 5.0 & 27 & 4.8 & 2 & 2.0 & 161 & 5.5 & $<\mathbf{0 . 0 0 1}$ \\
\hline Bulimia nervosa & 6 & 2.8 & 27 & 2.8 & $\mathrm{Y}$ & $\mathrm{Y}$ & 56 & 7.5 & 26 & 8.1 & 26 & 4.6 & 4 & 1.1 & 145 & 5.0 & $<0.001$ \\
\hline Body dysmorphic disorder & 8 & 3.7 & 111 & 11.6 & $\mathrm{Y}$ & $\mathrm{Y}$ & 179 & 23.9 & 42 & 13.0 & 36 & 6.4 & 6 & 6.1 & 382 & 13.1 & $<\mathbf{0 . 0 0 1}$ \\
\hline $\begin{array}{l}\text { Obsessive-compulsive } \\
\text { personality disorder }\end{array}$ & $\mathrm{Y}$ & $\mathrm{Y}$ & $\mathrm{Y}$ & $\mathrm{Y}$ & $\mathrm{Y}$ & Y & 334 & 44.5 & $\mathrm{Y}$ & $\mathrm{Y}$ & $\mathrm{Y}$ & $\mathrm{Y}$ & $\mathrm{Y}$ & $\mathrm{Y}$ & 334 & 44.5 & $<\mathbf{0 . 0 0 1}$ \\
\hline Suicide attempt & 41 & 18.7 & 97 & 10.2 & 62 & 7.7 & 17 & 2.3 & 44 & 13.7 & 90 & 15.9 & 4 & 4.1 & 314 & 9.0 & $<\mathbf{0 . 0 0 1}$ \\
\hline
\end{tabular}

$\mathrm{X}=$ Study exclusion criteria prevented participants having this co-occurring diagnosis. $\mathrm{Y}=$ Not assessed with diagnostic interview in this particular study.

${ }^{\text {a }}$ Data were missing for $31(14.2 \%)$ patients.

Table 4

Mean ages of onset of obsessive-compulsive disorder and co-occurring diagnoses across the samples from four nations $(\mathrm{N}=2022)$.

\begin{tabular}{|c|c|c|c|c|c|c|c|c|c|c|c|}
\hline & \multicolumn{2}{|l|}{$\begin{array}{l}\text { Australia } \\
(\mathrm{N}=219)\end{array}$} & \multicolumn{2}{|c|}{$\begin{array}{c}\text { Brazil } \\
(\mathrm{N}=955)\end{array}$} & \multicolumn{2}{|c|}{$\begin{array}{c}\text { Italy } \\
(\mathrm{N}=750)\end{array}$} & \multicolumn{2}{|l|}{$\begin{array}{c}\text { Spain } \\
(\mathrm{N}=98)\end{array}$} & \multicolumn{2}{|c|}{$\begin{array}{l}\text { Mean of } 4 \text { nations } \\
\quad(N=2022)\end{array}$} & \multirow{2}{*}{$\frac{\text { P-value }}{\text { Heterogeneity }}$} \\
\hline & Mean (years) & SD & Mean (years) & SD & Mean (years) & SD & Mean (years) & SD & Mean (years) & SD & \\
\hline Obsessive-compulsive disorder & 18.4 & 11.4 & 12.7 & 7.3 & 19.0 & 9.0 & 18.59 & 10.0 & 16.9 & 4.5 & 0.90 \\
\hline Major depressive disorder & 29.0 & 17.2 & 34.4 & 17.1 & 24 & 7.0 & 24.0 & 3.6 & 24.5 & 3.1 & 0.93 \\
\hline Dysthymia & 22.7 & 11.8 & 26.0 & 13.8 & 19.0 & 3.0 & 20.3 & 4.5 & 19.7 & 2.4 & 0.95 \\
\hline Manic episode & 19.6 & 15.5 & 25.0 & 22.6 & 25.0 & 4.0 & 20.1 & 2.5 & 21.5 & 2.1 & 0.77 \\
\hline Hypomanic episode & 20.4 & 8.5 & 23.5 & 9.5 & 30.0 & 7.0 & 18.3 & 2.1 & 19.5 & 1.9 & 0.43 \\
\hline Panic disorder & 24.2 & 13.0 & 28.5 & 12.9 & 28.0 & 5.0 & 21.1 & 4.6 & 24.5 & 3.2 & 0.77 \\
\hline Agoraphobia & 25.1 & 12.4 & 22.3 & 13.8 & 28.0 & 5.0 & 22.2 & 3.3 & 24.0 & 2.6 & 0.81 \\
\hline Social phobia & 19.0 & 13.4 & 18.4 & 12.5 & 20.0 & 10.0 & 17.1 & 2.1 & 17.3 & 2.0 & 0.99 \\
\hline Posttraumatic stress disorder & 28.0 & 16.6 & 24.3 & 14.9 & 22.0 & 11.0 & 25 & $\mathrm{a}$ & 24.0 & 7.8 & 0.96 \\
\hline Generalized anxiety disorder & 22.3 & 16.9 & 26.4 & 14.7 & 25.0 & 12.0 & 19.2 & 4.6 & 20.6 & 4.0 & 0.94 \\
\hline Specific phobia & 18.8 & 13.7 & 20.7 & 11.4 & 27.0 & 4.0 & 12.3 & 2.5 & 16.6 & 2.1 & 0.020 \\
\hline Alcohol abuse & 23.5 & 7.7 & 20.5 & 6.1 & 32.0 & 4.0 & 17.2 & 1.1 & 18.4 & 1.0 & 0.0040 \\
\hline Alcohol dependence & 27.1 & 11.6 & 24.4 & 4.3 & 35.0 & 9.0 & $\mathrm{X}$ & $\mathrm{X}$ & 26.4 & 3.7 & 0.57 \\
\hline Non-alcohol drug abuse & 19.2 & 5.7 & $X$ & $\mathrm{X}$ & 24.0 & 6.0 & 21 & a & 21.5 & 4.1 & 0.56 \\
\hline Non-alcohol drug dependence & 21.6 & 7.0 & $\mathrm{X}$ & $\mathrm{X}$ & 24.0 & 6.0 & $\mathrm{X}$ & $\mathrm{X}$ & 23.0 & 4.6 & 0.79 \\
\hline Psychotic disorder & 20.8 & 9.5 & 23.5 & 9.5 & 25.0 & 19.0 & $\mathrm{X}$ & $\mathrm{X}$ & 16.4 & 1.2 & 0.80 \\
\hline Hypochondriasis & 19.1 & 12.8 & 20.4 & 11.1 & 29.0 & 10.0 & 15.2 & 3.5 & 17.1 & 3.1 & 0.61 \\
\hline Anorexia nervosa & 18.2 & 6.3 & 20.3 & 10.1 & 22.0 & 4.0 & 14.0 & 1.2 & 14.8 & 1.1 & 0.23 \\
\hline Bulimia nervosa & 15.1 & 6.9 & 21.7 & 9.9 & 25.0 & 4.0 & 18.2 & 2.4 & 19.7 & 1.9 & 0.45 \\
\hline Body dysmorphic disorder & 13.8 & 4.9 & 17.9 & 10.3 & 28.0 & 5.0 & 16.5 & 2.4 & 17.9 & 1.9 & 0.16 \\
\hline
\end{tabular}

$\mathrm{X}=$ Study exclusion criteria prevented participants having this co-occurring diagnosis.

${ }^{\text {a }}$ Data came from only one patient. 


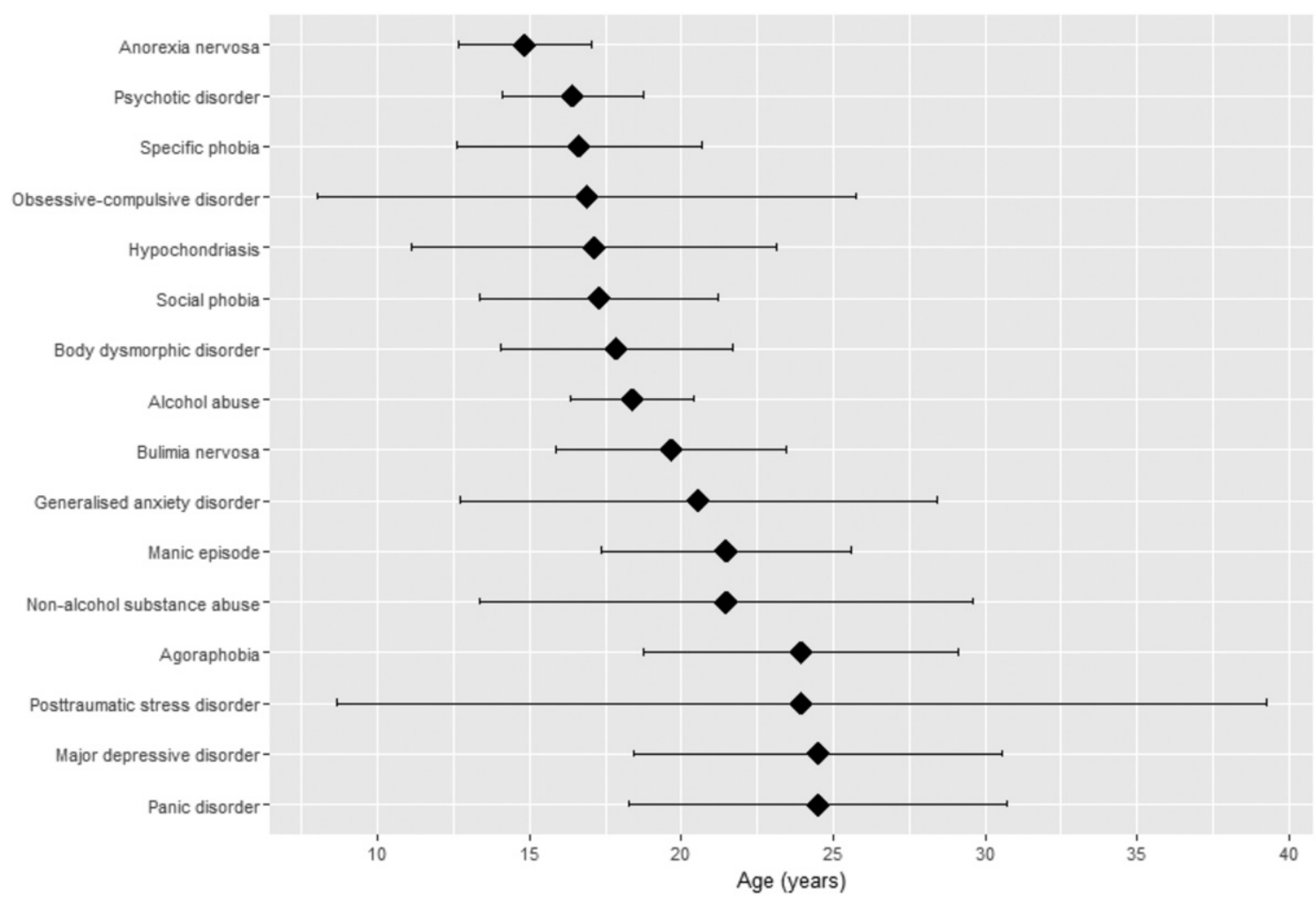

Fig. 1. Forrest plot of the ages of onset of obsessive-compulsive disorder and co-occurring disorders.

important treatment implications in terms of the need to address co-occurring depression in the course of treating OCD. Future studies should attempt to adjust for demographic variables such as age and use prospective design to establish the direction of a causal relationship, if any, between OCD and depressive disorders.

The relationship between OCPD and OCD has been discussed in the literature and has been viewed as complicated by overlapping diagnostic criteria [24,25]. OCPD was not assessed for in all studies and this might relate to difficulties assessing for comorbid personality disorders. The lower rates of co-occurring current OCPD in Spain $(8.2 \%, \mathrm{n}=8)$ and Japan $(14.0 \%, \mathrm{n}=45)$ require further investigation as one could speculate that cultural factors such as collective attitudes and levels of social acceptance for certain personality traits might influence these rates.

The three anxiety disorders that most frequently co-occurred with OCD as current and lifetime diagnoses were generalized anxiety disorder, specific phobia and social phobia. This finding is consistent with previous studies e.g. $[2,5]$ and supports an important relationship between these anxiety disorders and OCD. Specific phobia and social phobia had earlier ages of onset than generalized anxiety disorder. Yaryura-Tobias et al. (2000) suggest that specific phobia may have an early age of onset in patients with OCD. A recent prospective study demonstrated that specific phobia predicted the subsequent onset of a range of disorders, including OCD [26]. The World Health Organization's Mental Health Survey also found that phobias and separation anxiety had earlier ages of onset than generalized anxiety disorder, panic disorder and posttraumatic stress disorder and suggested two sets of ages of onset for anxiety disorders [27]. Hence it would be important to attempt to understand the nature of this association.

Depressive and anxiety disorders appeared to be more frequently comorbid with OCD than other DSM5 obsessivecompulsive and related disorders, such as hoarding disorder, body dysmorphic disorder, trichotillomania or skin-picking disorder. However, the findings were limited in that not all sites assessed for these disorders, nor did they assess for these disorders in a standardized way. Prospective studies assessing the ages of onset of these disorders might be useful in understanding how these disorders might be related.

Suicidal ideation within the last month was not rare. Consistent with more recent reports $[9,13,15,16]$, previous suicide attempts were also relatively frequent. In a previous study by de la Cruz et al. (2016), even when comorbidity was adjusted for, OCD alone emerged as an independent predictor of suicide risk. It remains uncertain whether findings of the current study are related to the presence of co-occurring psychiatric disorders that elevate the risk of suicidality, such as major depressive disorder, because we were unable to control for comorbidity. The considerable 
rates of suicidality in this study emphasize the need to assess for suicidality in patients with OCD and to monitor this risk.

The present study has a number of strengths and limitations. A strength of this study is that data were collected from specialized centers in which all patients received a diagnosis of OCD with clinician-administered diagnostic interviews and all were assessed for the severity of OCD by means of the Y-BOCS. Limitations include the fact that the participation of international centers from the five continents required a simple procedure, which did not allow all the relevant information to be recorded. Information was not available for all psychiatric diagnoses; for example, proposed obsessive-compulsive spectrum disorders such as trichotillomania and skin picking disorder were not assessed in a standardized manner at all sites. It should be noted that the age of onset of OCD may have differed between samples as it is often difficult to determine whether a participant's obsessive-compulsive symptoms in childhood are associated with functional decline (and thus meeting diagnostic criteria for OCD) or not. Data have been derived from studies with different objectives and different recruitment methods, making the samples from seven countries heterogeneous. This is particularly relevant when trying to understand the differences in rates of comorbidity between countries. Future studies should include standardized recruitment procedures and adjust for variables such as OCD severity, age and gender so that variation across different countries can be better understood. It may then be useful to further evaluate cultural and social factors such as mental health literacy and early detection and their association with rates of comorbid diagnoses. If specific cultural or social factors are suspected, these factors may serve as useful prevention strategies.

\subsection{Conclusion}

This study involving a large sample and several international centers provides further evidence of the high rates of psychiatric comorbidity in OCD. It also highlights the prevalence of suicidal ideation and suicide attempts in patients with OCD and the need for clinicians to assess and monitor suicide risk. Consistent with community surveys (e.g. Kessler et al., 2007), the age of onset of co-occurring specific phobia and social phobia was early, whereas the onset of co-occurring generalized anxiety disorder and major depressive disorder was late in comparison to the onset of OCD. This finding needs to be explored with well-designed prospective studies that also assess other DSM5 obsessivecompulsive and related disorders and that aim to explore how these disorders may be etiologically related.

\section{References}

[1] Brakoulias V, Starcevic V, Berle D, Sammut P, Milicevic D, Moses K, et al. Further support for five dimensions of obsessive-compulsive symptoms. J Nerv Ment Dis 2013;201(6):452-9.
[2] Ruscio A, Stein D, Chiu W, Kessler R. The epidemiology of obsessive-compulsive disorder in the National Comorbidity Survey Replication. Mol Psychiatry 2010;15(1):53-63.

[3] Kalra H, Trivedi JK, Dalal PK, Sinha PK, Allet JL. Uncomplicated and complicated obsessive-compulsive disorder: an exploratory study from India. Compr Psychiatry 2008;49(1):51-4.

[4] Tukel R, Polat A, Ozdemir O, Aksut D, Turksoy N. Comorbid conditions in obsessive-compulsive disorder. Compr Psychiatry 2002;43(3):204-9.

[5] Torres AR, Fontenelle LF, Shavitt RG, Ferrao YA, do Rosario MC, Storch EA, et al. Comorbidity variation in patients with obsessivecompulsive disorder according to symptom dimensions: results from a large multicentre clinical sample. J Affect Disord 2016;190:508-16.

[6] Lochner C, Fineberg NA, Zohar J, Van Ameringen M, Juven-Wetzler A, Altamura AC, et al. Comorbidity in obsessive-compulsive disorder (OCD): a report from the International College of ObsessiveCompulsive Spectrum Disorders (ICOCS). Compr Psychiatry 2014;55(7):1513-9.

[7] Yaryura-Tobias JA, Grunes MS, Todaro J, McKay D, Neziroglu FA, Stockman R. Nosological insertion of axis I disorders in the etiology of obsessive-compulsive disorder. J Anxiety Disord 2000;14(1):19-30.

[8] de Mathis MA, Diniz JB, Hounie AG, Shavitt RG, Fossaluza V, Ferrao Y, et al. Trajectory in obsessive-compulsive disorder comorbidities. Eur Neuropsychopharmacol 2013;23(7):594-601.

[9] de la Cruz LF, Rydell M, Runeson B, D’Onofrio B, Brander G, Rück $\mathrm{C}$, et al. Suicide in obsessive-compulsive disorder: a population-based study of 36788 Swedish patients. Mol Psychiatry 2016.

[10] Kanwar A, Malik S, Prokop LJ, Sim LA, Feldstein D, Wang Z, et al. The association between anxiety disorders and suicidal behaviors: a systematic review and meta-analysis. Depress Anxiety 2013;30(10):917-29.

[11] Torres AR, de Abreu Ramos-Cerqueira AT, Torresan RC, de Souza Domingues M, Hercos ACR, Guimaraes ABC. Prevalence and associated factors for suicidal ideation and behaviors in obsessivecompulsive disorder. CNS Spectr 2007;12(10):771-8.

[12] Fawzy N, Hashim H. Prevalence and risk factors of suicide among patients with obsessive-compulsive disorder. Middle East Curr Psychiatry 2011;18(1):18-22.

[13] Angelakis I, Gooding P, Tarrier N, Panagioti M. Suicidality in obsessive compulsive disorder (OCD): a systematic review and metaanalysis. Clin Psychol Rev 2015;39:1-15.

[14] Kamath P, Reddy YJ, Kandavel T. Suicidal behavior in obsessivecompulsive disorder. J Clin Psychiatry 2007;68(11):1741-50.

[15] Torres AR, Ramos-Cerqueira ATA, Ferrão YA, Fontenelle LF, do Rosário MC, Miguel EC. Suicidality in obsessive-compulsive disorder: prevalence and relation to symptom dimensions and comorbid conditions [CME]. J Clin Psychiatry 2011;72(1):17-26.

[16] Velloso P, Piccinato C, Ferrão Y, Perin EA, Cesar R, Fontenelle L, et al. The suicidality continuum in a large sample of obsessivecompulsive disorder (OCD) patients. Eur Psychiatry 2016;38:1-7.

[17] De Berardis D, Serroni N, Campanella D, Rapini G, Olivieri L, Feliziani B, et al. Alexithymia, responsibility attitudes and suicide ideation among outpatients with obsessive-compulsive disorder: an exploratory study. Compr Psychiatry 2015;58:82-7.

[18] Miguel EC, Ferrão YA, Rosário MCd, Mathis MAd, Torres AR, Fontenelle LF, et al. The Brazilian Research Consortium on Obsessive-Compulsive Spectrum Disorders: recruitment, assessment instruments, methods for the development of multicenter collaborative studies and preliminary results. Rev Bras Psiquiatr 2008;30(3):185-96.

[19] Goodman WK, Price LH, Rasmussen SA, Mazure C, Fleischmann RL, Hill CL, et al. The Yale-Brown Obsessive Compulsive Scale. I. Development, use, and reliability. Arch Gen Psychiatry 1989;46(11):1006-11.

[20] First MB, Spitzer RL, Gibbon M, Williams JB. SCID-I/P; 2007.

[21] Sheehan DV, Lecrubier Y, Sheehan KH, Amorim P, Janavs J, Weiller E, et al. The Mini-International Neuropsychiatric Interview (M.I.N.I.): the development and validation of a structured diagnostic psychiatric 
interview for DSM-IV and ICD-10. J Clin Psychiatry 1998;59(Suppl 20):22-33 [quiz 4-57].

[22] Sheehan DV, Lecrubier Y. MINI International Neuropsychiatric Interview English version 6.0.0 2010. [cited 2013 23.01.13]. Available from: http://rucklab.files.wordpress.com/2012/03/mini-6-0oct-10-2010.pdf.

[23] Brown TA, Barlow DH, Di Nardo PA. Anxiety disorders interview schedule adult version: client interview schedule. Oxford University Press; 1994.

[24] Starcevic V, Brakoulias V. New diagnostic perspectives on obsessivecompulsive personality disorder and its links with other conditions. Curr Opin Psychiatry 2014;27(1):62-7.
[25] Lochner C, Serebro P, van der Merwe L, Hemmings S, Kinnear C, Seedat S, et al. Comorbid obsessive-compulsive personality disorder in obsessive-compulsive disorder (OCD): a marker of severity. Prog Neuropsychopharmacol Biol Psychiatry 2011;35(4):1087-92.

[26] Lieb R, Miché M, Gloster AT, Beesdo-Baum K, Meyer AH, Wittchen HU. Impact of specific phobia on the risk of onset of mental disorders: a 10-year prospective-longitudinal community study of adolescents and young adults. Depress Anxiety 2016;33(7):667-75.

[27] Kessler RC, Matthias A, Anthony JC, De Graaf R, Demyttenaere K, Gasquet I, et al. Lifetime prevalence and age-of-onset distributions of mental disorders in the World Health Organization's World Mental Health Survey Initiative; 2007. 